\title{
Surface Normal Lasing of CdSe Nanoplatelets Coupled to Aluminum Plasmonic Nanoparticle Lattices
}

Nicolas E. Watkins, ${ }^{1}$ Jun Guan, ${ }^{1}$ Benjamin T. Diroll, ${ }^{2}$ Kali R. Williams, ${ }^{1}$ Richard D. Schaller, ${ }^{1,2}$ and Teri W. Odom ${ }^{1,3 *}$

${ }^{1}$ Department of Chemistry, ${ }^{3}$ Department of Materials Science and Engineering, Northwestern University, Evanston, Illinois, 60208, USA

${ }^{2}$ Center for Nanoscale Materials, Argonne National Laboratory, Lemont, Illinois 60439, USA *email: todom@,northwestern.edu

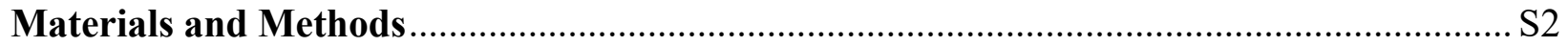

Measured Transmission from CdSe NPL Film Without Al NP array ............................. S5

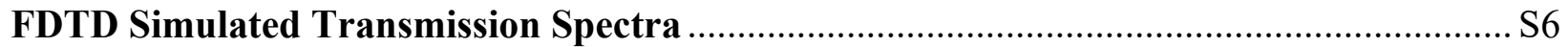

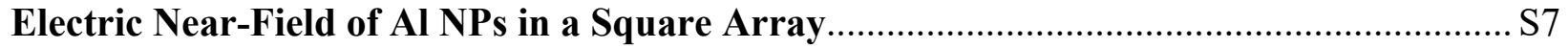

Scanning Electron Microscopy Images of Array Fabrication ........................................ S8

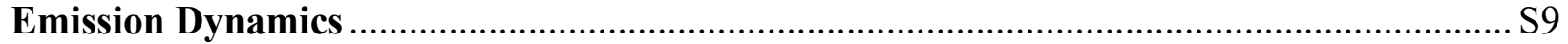

Atomic Force Microscopy Characterization of Al NP Height ....................................... S10

Atomic Force Microscopy Characterization of NPL Film Thickness............................. S1

Average Roughness of Drop-Casted NPL Film............................................................ S12

Angle-Resolved Amplified Spontaneous Emission ..................................................... S13

Emission Measured from CdSe NPL Thin Film on Al NP Array ................................... S14

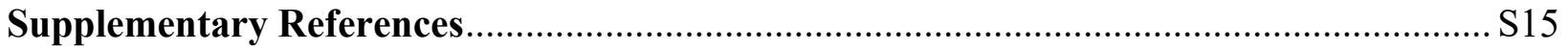




\section{Materials and Methods}

Chemicals: Commercially available reagents were purchased from Sigma Aldrich and used without requiring additional purification steps. Cadmium nitrate tetrahydrate (98\%), sodium myristate $(\geq 99 \%)$, selenium powder $(\geq 99.5 \%)$, cadmium acetate dihydrate $(98 \%)$, oleic acid (90\%), 1-octadecene (90\%), octane (98\%), methanol ( $\geq 99.5 \%)$, n-hexane.

5-Monolayer (ML) CdSe Nanoplatelet (NPL) Synthesis: Cadmium myristate precursor was prepared using a previously reported synthesis. ${ }^{1}$ A mortar and pestle were used to grind the dried precipitate into a fine powder used in the nanoplatelet synthesis.

5 ML CdSe NPLs were synthesized according to previously published procedures with minor modifications. ${ }^{1,2} 170 \mathrm{mg}$ of cadmium myristate and $14 \mathrm{~mL}$ of octadecene were placed in a $25 \mathrm{~mL}$ three-neck flask and degassed for 30 - 60 minutes using a roughing pump. The flask was then heated under nitrogen flow to $250^{\circ} \mathrm{C}$, at which point $1 \mathrm{~mL}$ of selenium powder that was sonicated in octadecene $(12 \mathrm{mg} / \mathrm{mL})$ for five minutes was rapidly injected. After $60 \mathrm{~s}, 100 \mathrm{mg}$ of finelyground cadmium acetate was added to the reaction flask, which was maintained at $250{ }^{\circ} \mathrm{C}$ for 10 min. The reaction was then allowed to cool; when the temperature reached $100{ }^{\circ} \mathrm{C}, 2 \mathrm{~mL}$ of oleic acid was added, and hexanes were then used to suspend the NPLs. The mixture was centrifuged at 14,500 RPM for $3 \mathrm{~min}$, and the supernatant was discarded. The resulting pellet was dispersed in hexane and filtered through a $0.2 \mu \mathrm{m}$ PTFE syringe filter.

FDTD simulations of lasing and ASE: FDTD calculations based on commercial software (FDTD Solution, Lumerical Inc., Vancouver, Canada) were used to simulate the linear transmittance of Al NP lattices. The optical constants of Al were taken from Palik (400-1000 nm). ${ }^{2}$ A uniform mesh size of $2 \mathrm{~nm}$ ( $\mathrm{x}, \mathrm{y}$ and $\mathrm{z}$ directions) was used. We set perfectly matched layer (PML) boundary conditions for the $\mathrm{z}$ direction, and periodic boundary conditions for $\mathrm{x}$ and $\mathrm{y}$ 
directions of the simulation region. We then represented the CdSe gain layer using a 110-nm thick rectangular slab with refractive index $n=1.8$. To simulate off-normal transmission spectra, we using Bloch boundary conditions for both the $\mathrm{x}$ and $\mathrm{y}$ directions. We then adjusted the sweeping angle of incident light $\left(0-2^{\circ}\right)$ to observe the $( \pm 1,0)$ and $(0, \pm 1) \mathrm{W}_{\text {TE-SLR }}$ modes respectively.

Fabrication of nanoparticle arrays: A poly(dimethylsiloxane) mask with square lattice spacing $a_{0}=400 \mathrm{~nm}$ was used to pattern photoresist posts on $\mathrm{Si}$ (100) wafers coated in Shipley S1805 photoresist using solvent-assisted nanoscale embossing. ${ }^{3}$ Reactive ion etching was then used to remove resist residue using oxygen plasma. An array of holes was created by thermally evaporating a thin layer of $\mathrm{Cr}(10 \mathrm{~nm})$ and lifting-off the photoresist posts using Microposit ${ }^{\mathrm{TM}}$ developer. Then deep reactive ion etching was used to create cylindrical pits into the Si beneath the circular Cr holes. Au nanohole arrays with a smaller diameter $d=100 \mathrm{~nm}$ were then produced by depositing 150-nm Au followed by etching the $\mathrm{Cr}$ sacrificial layer. Au nanohole arrays were floated onto substrates as deposition masks that are readily removed by Scotch tape after metal deposition. An electron-beam evaporator (AJA) was used to deposit $2 \mathrm{~nm}$ of $\mathrm{Cr}$ (adhesion layer) followed by $60 \mathrm{~nm}$ of Al through the Au holes. Scotch tape was then softly pressed onto the hole mask and removed.

Preparing Drop-cast Dispersions and Films: $2.5 \mathrm{~mL}$ of dispersion, prepared as described above, with optical density $=2.0(1 \mathrm{~mm}$ pathlength cuvette $)$ was added to a centrifuge tube and spun at 14,500 RPM. The supernatant was discarded and the NPL pellet was lightly dried under nitrogen to evaporate remaining solvent. Using the mass of the centrifuge tube, the mass of the NPL pellet was back calculated and found to be $\sim 7.3 \mathrm{mg}$. The pellet was dispersed using $35 \mu \mathrm{L}$ of a hexane:octane solvent mixture (9:1 ratio). Different ratios of hexane and octane were tested to achieve smooth CdSe NPL films. We found that this solvent combination prevented films from 
spreading to the far edge of the substrate and produced a coffee-ring effect of increasing thickness near the edge of the film, which enabled us to study varying thicknesses across the Al NP array.

Lasing measurements: CdSe NPL films that were drop-cast on Al NP lattices were pumped by a regeneratively amplified Ti:sapphire laser $(800 \mathrm{~nm}$ wavelength, $1000 \mathrm{~Hz}$ repetition rate, and 35 fs pulse width) that was passed through a beta barium borate doubling crystal to produce 400nm light. Samples were excited at an incident angle of $\sim 45^{\circ}$ and lasing signals were collected normal to the sample surface and directed to a $0.3 \mathrm{~m}$ spectrometer and CCD. The laser fluence was adjusted using a neutral density filter wheel and a power meter. For angle-resolved emission measurements, the sample was fixed and angle-dependent emission signals were collected using a fiber optic mounted to a rotational stage.

Time-resolved emission dynamics: Time-correlated single photon counting (TCSPC) and a streak camera were both used to measure fluence-dependent, wavelength-resolved PL decay experiments to characterize emission timescales. A $1000 \mathrm{~Hz}$ amplified Ti:sapphire laser doubled to $400 \mathrm{~nm}$ was used to photoexcite samples at an incident angle of $45^{\circ}$. The emission signals were passed through a neutral density filter $(\mathrm{ND}=1.0)$, and the dispersed emission was detected with a single-photon sensitive streak camera. Streak camera measurements were necessary to collect the spectrally resolved dynamics of lasing emission and also to provide higher time-resolution (2 ps) in addition to analog photon collection. TCSPC ( $50 \mathrm{ps}$ resolution) was used to measure the longlived single-exciton radiative recombination, which is on the order of nanoseconds. 


\section{Measured Transmission from CdSe NPL Film Without Al NP array}

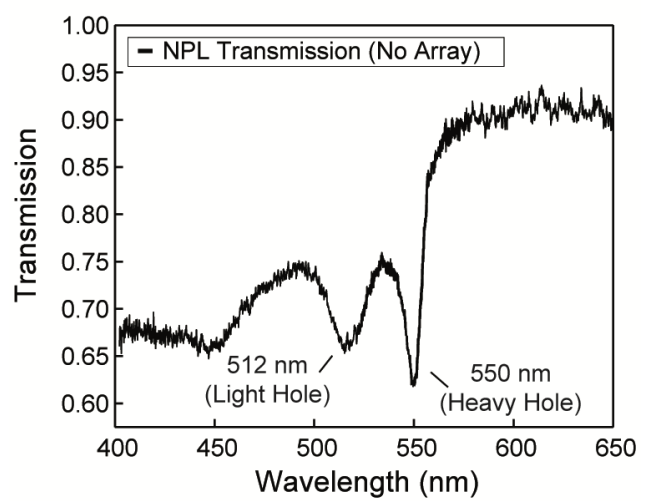

Figure S1: CdSe NPL film transmission without Al NP lattice. Measured transmission of 5-monolayer CdSe NPL under white-light excitation. Film thickness is approximately 100 $\mathrm{nm}$. 


\section{FDTD Simulated Transmission Spectra}
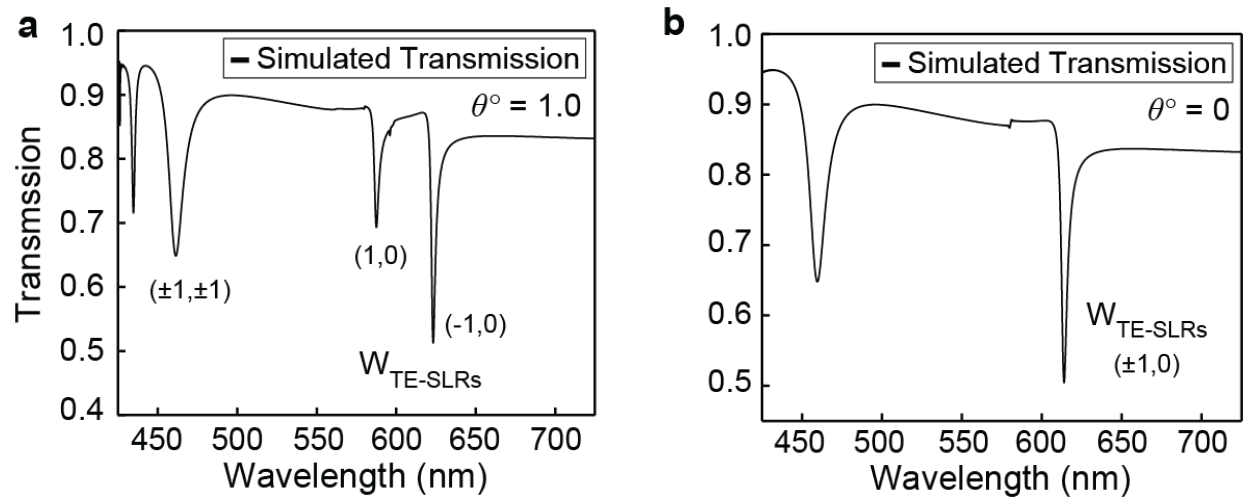

Figure S2: W-SLR transmission simulated using FDTD. Simulated transmission spectra of an Al NP lattice with CdSe NPL film at incident angle of (a) $1^{\circ}$ and (b) $0^{\circ}$. The CdSe gain layer was generated using a rectuangular slab with a thickness of $110 \mathrm{~nm}$ and $n=1.8$. The spectrum at $1^{\circ}$ revealed two $\mathrm{W}_{\text {TE-SLR }}$ modes that agree with the experimentally measured spectrum. A higher order mode $( \pm 1, \pm 1)$ can be seen at bluer wavelengths in simulation but was not observed in experiment. 
Electric Near-Field of Al NPs in a Square Array

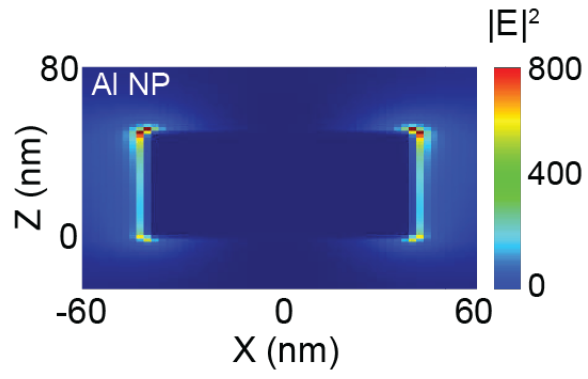

Figure S3: Calculated Electric Field Distributions for an Al NP array. Electric field

distribution for a Al NP array with a square lattice geometry with diameter $=70 \mathrm{~nm}$, height $=$ $60 \mathrm{~nm}$, and high-index NPL film superstrate (100 nm, $n=1.8)$. 
Scanning Electron Microscopy Images of Lattice Fabrication

a

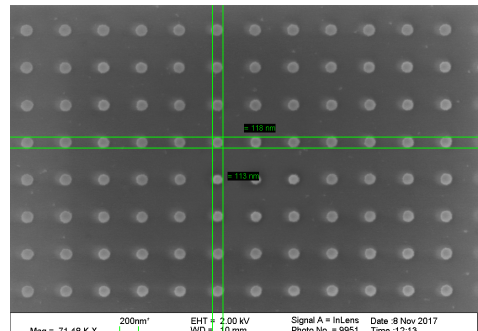

d

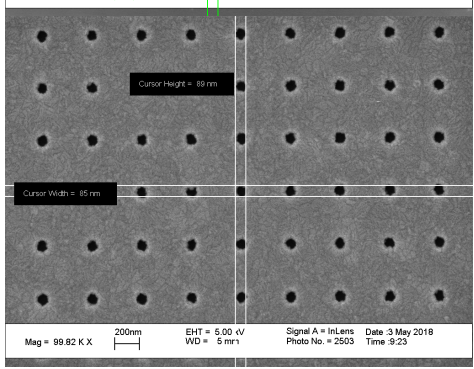

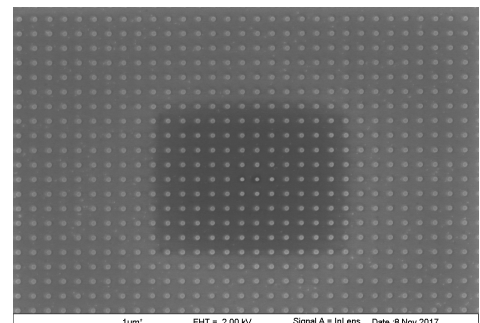

e

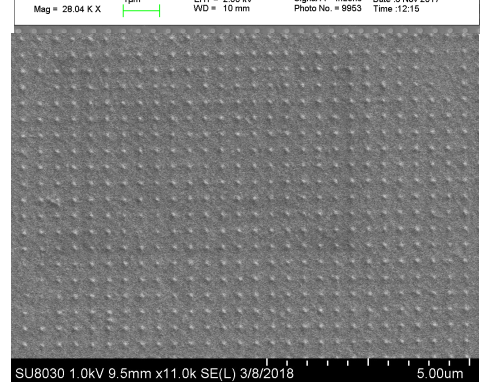

C
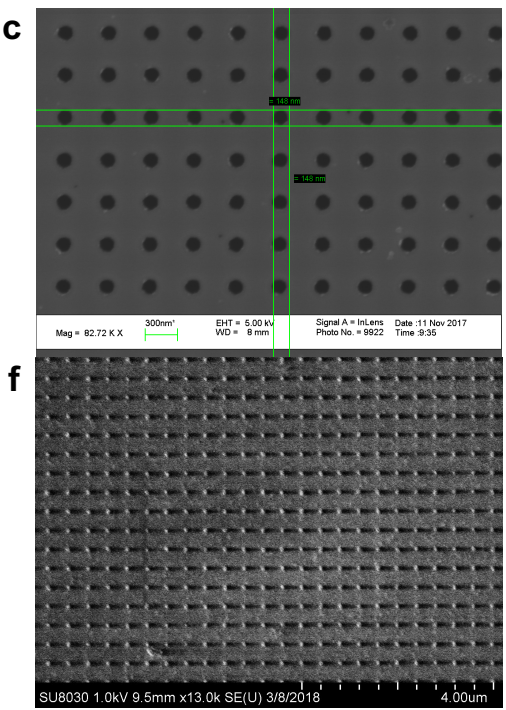

Figure S4: SEM Characterization of NP lattice fabrication. (a-b) Photoresist posts after reactive ion etching. (b) Cr hole array after deep reactive ion etching. (d) Gold hole array after depositing onto Cr hole array. Array periodicity $\left(a_{0}=400 \mathrm{~nm}\right)$. (e-f) Al nanoparticle lattice on quartz substrate. 


\section{Emission Dynamics}

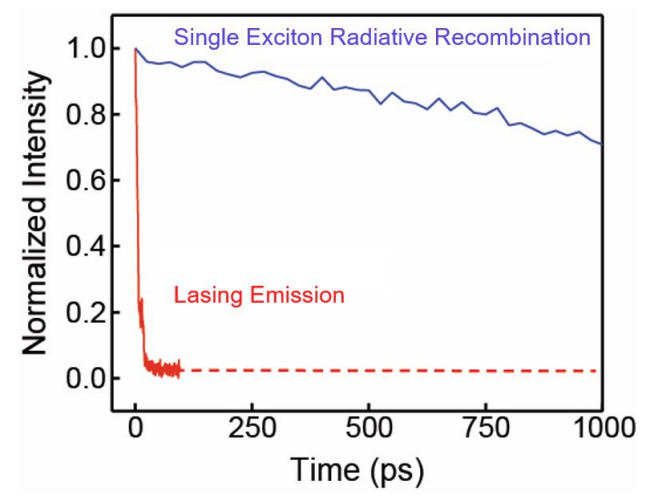

Figure S5: Emission dynamics of CdSe nanoplatelet PL vs. lasing. Single exciton radiative recombination without array (blue trace, off array) and lasing emission at $577 \mathrm{~nm}$ (red trace, on array), collected with time correlated single photon counting (TCSPC) and streak camera respectively. TCSPC was collected with a fluence $\left(95 \mu \mathrm{J} / \mathrm{cm}^{2}\right)$ below nanoplatelet ASE threshold $\left(170 \mu \mathrm{J} / \mathrm{cm}^{2}\right)$, while streak camera data was collected above nanoplatelet ASE and lasing threshold $\left(200 \mu \mathrm{J} / \mathrm{cm}^{2}\right)$. 


\section{Atomic Force Microscopy Characterization of Al NP Height}
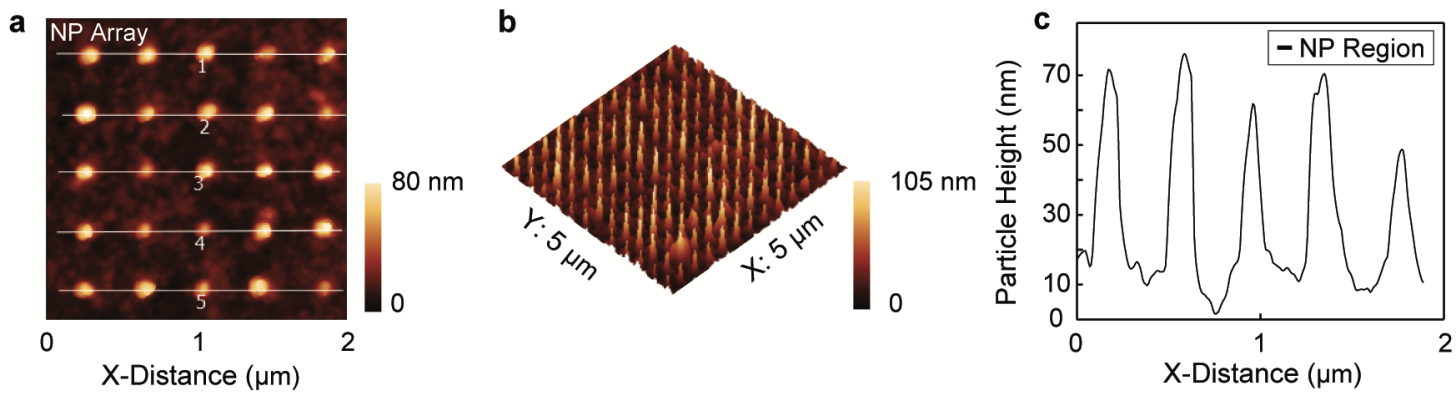

Figure S6: NP height determination by AFM. (a) AFM image of Al NPs in a region without CdSe NPL film. (b) AFM tilted image of 5 by $5 \mu \mathrm{m}$ patch of Al NPs. (c) Height profiles NPs in lattice with average height $=60 \mathrm{~nm}$. 


\section{Atomic Force Microscopy Characterization of NPL Film Thickness}
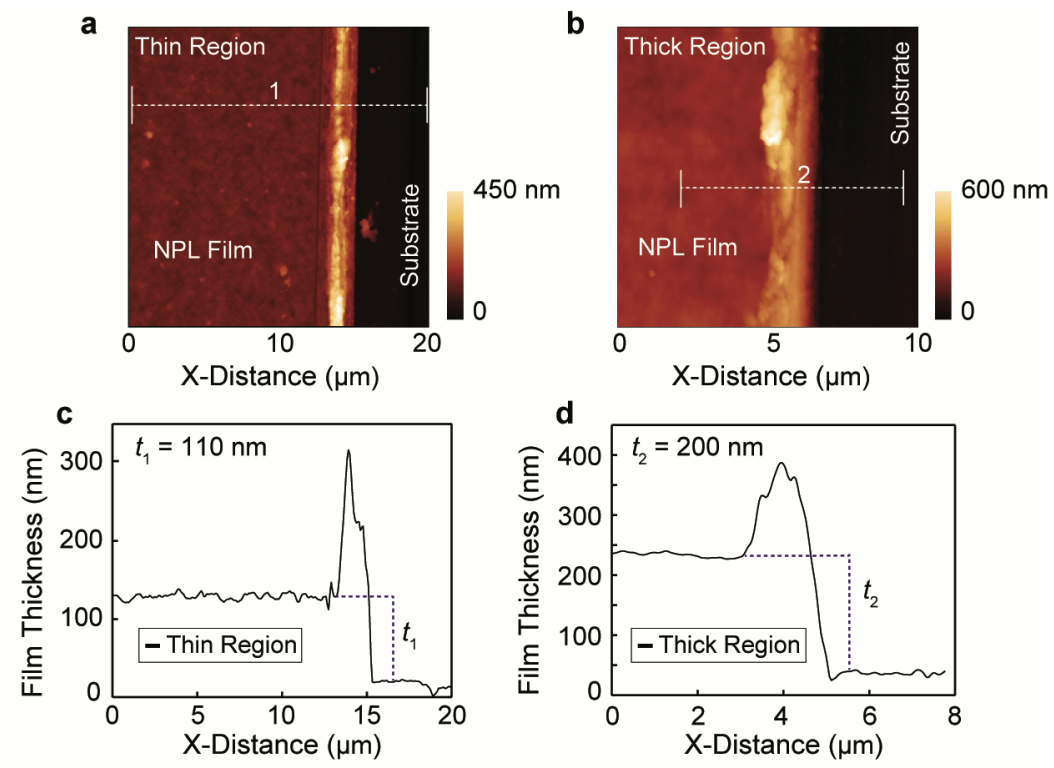

Figure S7: Determining NPL film thickness across NP lattice region using atomic force microscopy (AFM). (a-b) AFM images of representative thick and thin film regions in Figure 3a. Note: Bright region between film and substrate is from a scratch made for the measurement and was not included in the thickness calculation. (c-d) Calculated film thickness for respective regions using line profiles. 


\section{Average Roughness of Drop-Casted NPL Film}

a

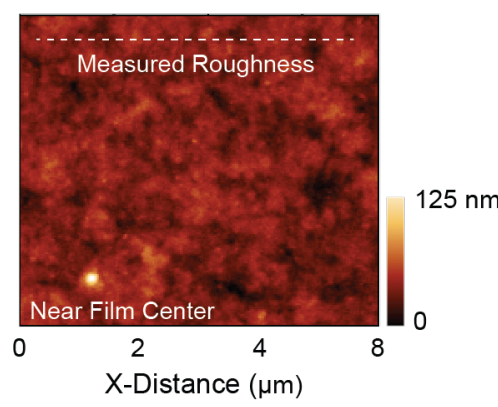

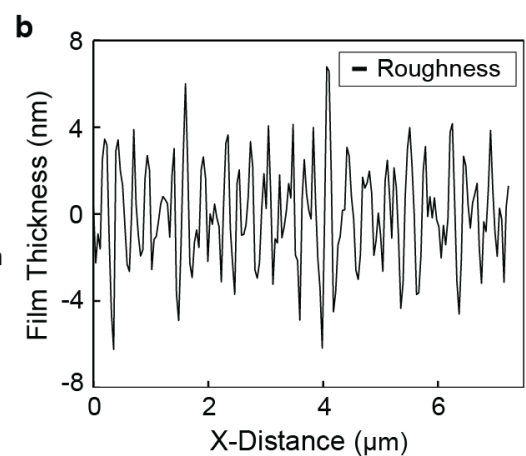

Figure S8: NPL film roughness near center region of NPL film. (a) AFM image of CdSe NPL film drop-cast on substrate. The dotted line represents the measured region. (b) Extracted one-dimensional roughness profile shows film is relatively smooth, with an average roughness $\left(\mathrm{R}_{\mathrm{a}}\right)=1.96 \mathrm{~nm}$ across $7 \mu \mathrm{m}$. The average roughness was calculated using the rootmean square method: $R=\frac{1}{N} \sum_{j=1}^{N}\left|r_{j}\right|$, where $r_{j}=z_{j}-\bar{z}$, where $\mathrm{R}$ is the average sum of all points from the evaluation length, $\mathrm{r}$ is roughness, and $\mathrm{z}$ is film height. 


\section{Angle-Resolved Amplified Spontaneous Emission}

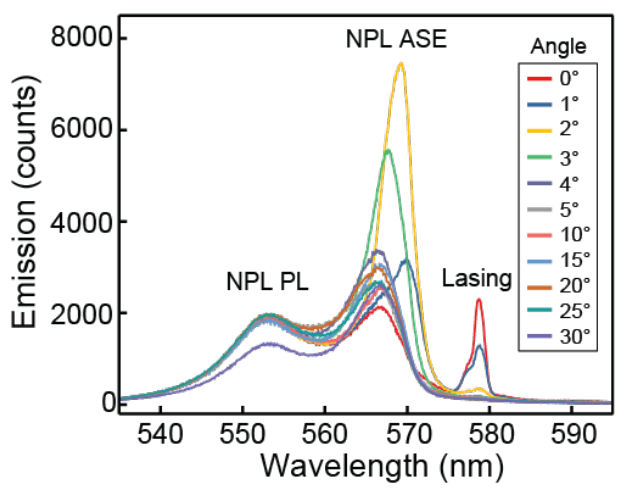

Figure S9: Angle-resolved emission from CdSe NPL and Al NP lattice. Angle-resolved emission collected as a function of detection angle using a fluence of $\sim 220 \mu \mathrm{J} / \mathrm{cm}^{2}$. In each case, lasing signals exhibited surface-normal directionality, while ASE signals can be observed for all angles measured. 


\section{Emission Measured from CdSe NPL Thin Film on Al NP Array}

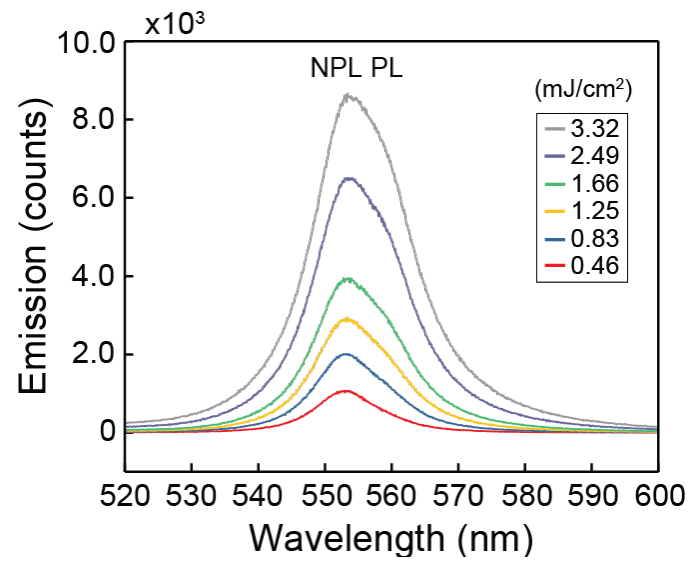

Figure S10: Emission from CdSe NPL film drop-cast from a dilute dispersion on a Al NP lattice. Emission from a thin $(<50 \mathrm{~nm})$ CdSe NPL film created from a ten-fold diluted concentrated dispersion $(\sim 20 \mathrm{mg} / \mathrm{mL})$. Some physical degradation after high pump powers was observed. 


\section{Supplementary References}

1. Ithurria, S.; Bousquet, G.; Dubertret, B., Continuous Transition from 3D to 1D Confinement Observed during the Formation of CdSe Nanoplatelets. Journal of the American Chemical Society 2011, 133 (9), 3070-3077.

2. Edited by Edward, D. P., Handbook of optical constants of solids. Orlando : Academic Press, 1985.: 1985.

3. Lee, M. H.; Huntington, M. D.; Zhou, W.; Yang, J.-C.; Odom, T. W., Programmable Soft Lithography: Solvent-Assisted Nanoscale Embossing. Nano Letters 2011, 11 (2), 311-315. 